\title{
ESTRÉS LABORAL EN TRABAJADORES DE LA CONSTRUCCIÓN
}

\section{Autor:}

César Augusto Chávez Orozco'

1Dirección General de Posgrados

Universidad Tecnológica Equinoccial

Correo electrónico: coca2550@ute.edu.ec

Dirección: Calle Rumipamba s/n, entre Burgeois y Av. Atahualpa

Teléfonos: (593) 2 299-0800 / Fax: (593) 2 244-2288

Quito - Ecuador 


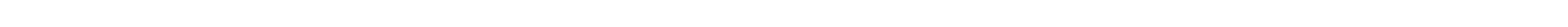




\section{RESUMEN}

La presente investigación pretende determinar la presencia de estrés laboral en trabajadores de la construcción y la industria; que al igual que otros tipos de industrias, presenta riesgos que pueden ocasionar enfermedades, lesiones, discapacidad y muertes en el trabajo, siendo los riesgos psicosociales uno de los más perjudiciales, como consecuencia de unas malas condiciones de trabajo, concretamente de una deficiente organización del trabajo, y que pueden ocasionar: estrés, enfermedades cardiovasculares, respiratorias, inmunitarias, gastrointestinales, dermatológicas, endocrinológicas, músculo esqueléticas y mentales.

Palabras clave: Estrés laboral, Factores psicosociales, Demanda control.

\section{ABSTRACT}

This research to determine the presence of occupational stress in construction workers and industry; like other industries, presents risks that could cause illness, injury, disability and deaths at work, psychosocial risks being one of the most damaging, as a result of poor working conditions, namely poor organization of work, and that can cause stress, cardiovascular, respiratory, immune, gastrointestinal, dermatological diseases, endocrine, skeletal and mental muscle.

Keywords: Job stress, psychosocial factors, Demand Control. 


\section{INTRODUCCIÓN}

El estrés laboral es un fenómeno personal y social cada vez más frecuente y con consecuencias importantes a nivel individual y organizacional. A nivel individual, afecta al bienestar físico, psicológico y la salud de las personas. A nivel colectivo afecta la salud organizacional.

Los trabajadores de la construcción realizan obras de diferente tipo y magnitud, como vías, puentes, edificios, estructuras, represas, sistemas de conducción de agua, parques, etc., así como también la reparación y el mantenimiento de la infraestructura física de un país. Al igual que en otros ramos de la industria, en el sector de la construcción también se encuentran presentes los riesgos laborales que causan enfermedades, lesiones, discapacidad y muertes relacionadas con el trabajo, siendo los riesgos psicosociales uno de ellos y en especial el estrés laboral.

El objetivo del presente estudio fue determinar el nivel de estrés relacionado con el trabajo en trabajadores de la construcción, mediante la aplicación del cuestionario de estrés denominado modelo demanda-control-apoyo social publicado por Karasek y Theorell en 1979. El estudio se realizó en el mes de septiembre de 2014 en la ciudad de Quito, en dos proyectos de construcción, con la participación de 150 trabajadores que se encontraban laborando en esos momentos, entre personal administrativo y obreros (bodegueros, albañiles, electricistas, etc.).

Se realizó un estudio de tipo descriptivo, exploratorio, con la participación del total de trabajadores; 130 (87\%) eran hombres y 20 (13\%) eran mujeres. El reactivo utilizado arrojó un factor de confiabilidad según el alfa de Cronbach de 0,908, y un coeficiente de confiabilidad rk20 de 0,89.

El estudio determinó que existe un bajo nivel de personas que ejecutan un trabajo de alta tensión laboral (23\%), y con riesgo de alta tensión psicológica y enfermedad física (Job stress); el personal femenino tiene un mayor riesgo de alta tensión laboral con $40 \%$. Los trabajadores con edad mayor a 50 años tiene el más alto grado de tensión psicosocial, con un 30,0\%; y existe un $7 \%$ de la población que se encuentra en la etapa de isostrain, con un alto riesgo de tensión mental y enfermedad física, este bajo nivel está relacionado con el alto nivel de apoyo social que existe por parte de las jefaturas y los compañeros.

\section{MÉTODOS Y MATERIALES}

Se realizó un estudio descriptivo, exploratorio, con la participación de 150 trabajadores de la construcción, en la ciudad de Quito. La encuesta se aplicó en el mes de septiembre de 2014, y participó personal administrativo y obreros; y se aplicó al 100\% de los trabajadores de dos proyectos que se encontraban en ese período de tiempo.
Para la recolección de datos se utilizó el cuestionario de estrés relacionado con el trabajo, denominado modelo de demandacontrol-apoyo social publicado por Karasek y Theorell en 1979, que consta de 29 preguntas y cuatro escalas de respuesta. Los datos se tabularon en Excel y el análisis se realizó en el programa SPSS. Este modelo identifica dos aspectos fundamentales en el ámbito del trabajo desencadenantes de estrés: la demanda y el control, y conceptualiza el estrés relacionado con el trabajo como la combinación de altas demandas psicológicas y escaso control en la toma de decisiones.

Este modelo de tensión de trabajo, establece que la combinación de altas demandas laborales y la baja capacidad de decisión conducen a resultados negativos en la salud física. Adicionalmente, el modelo contiene importantes predicciones sobre los rasgos de socialización de la personalidad y de patrones de comportamiento que ocurren en el trabajo. La adaptación a situaciones de bajas demandas y bajo control (trabajos pasivos) puede devenir en una reducción de la habilidad para resolver problemas o enfrentar retos, así como pueden aparecer sentimientos de depresión conocidos como "desesperanza aprendida" Figura 1 (Román, 2003, pág. 11).

Figura 1. Modelo demanda-control de Karasek. Fuente: Román 2003.

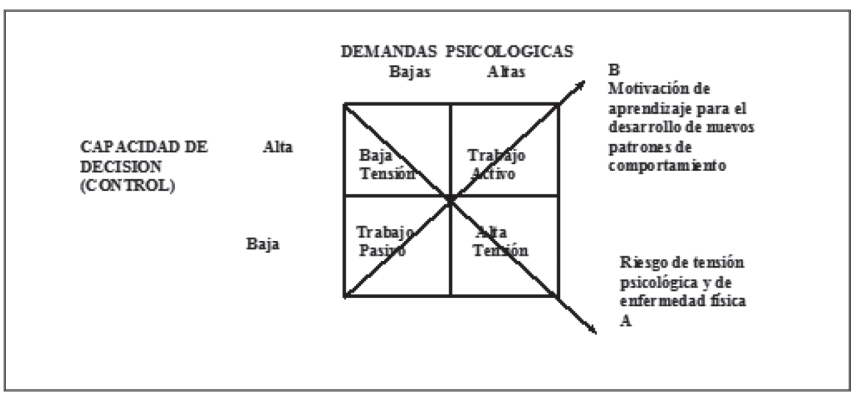

Las altas demandas son los elementos de estrés psicológico presentes en el ambiente laboral, como son la cantidad de trabajo a realizar, la ausencia de tiempo suficiente para realizar las tareas, la cantidad de trabajo que se realiza bajo presión del tiempo, el volumen de trabajo, el nivel de concentración requerido y la presencia de situaciones que obligan a interrumpir las ocupaciones para esperar que otros finalicen sus tareas (Martín, 2007).

El control se refiere a la capacidad que tienen los trabajadores sobre sus tareas y sobre la organización de su trabajo; y tiene dos componentes: el uso de habilidades, es decir, el grado en el que el trabajo implica aprender nuevas cosas, el estímulo de la creatividad y la posibilidad de desarrollar las propias capacidades; y la autoridad o aptitud en la toma de decisiones en el trabajo y su capacidad de influir en el grupo de trabajo (Martín, 2007). 
El modelo contempla la tercera dimensión, el apoyo social en el trabajo, que se refiere a la interacción de ayuda disponible en el lugar de trabajo, tanto por parte de los compañeros como de los superiores (Martín, 2007). El modelo indica que existe una asociación entre alteraciones en la salud y el estrés laboral, resultante de la combinación de las altas demandas psicológicas en el trabajo y la escasa capacidad de control sobre el mismo, dando lugar a alteraciones de la salud. De otra parte, el modelo añade que el apoyo social reduce el efecto del estrés. El método, describe cuatro tipos de trabajo (Román 2003):

Trabajos de alta tensión Laboral (TATL): Producto de la combinación de altas demandas y bajo control. Ésta es la combinación crítica del modelo. Se plantea que produce tensión psicofisiológica y reacciones adversas como fatiga, depresión, ansiedad, y eventuales trastornos físicos. El trabajo típico es la línea de ensamblaje.

Trabajos pasivos (TP): Son producto de la combinación de bajas demandas y bajo control. En tales trabajos, los trabajadores no suelen tener oportunidades para emplear sus habilidades. Combinados con la carencia de retos en el trabajo, pueden resultar en trabajos pocos motivantes con un riesgo promedio de tensión psicológica y de enfermedad. Un ejemplo de primera mano de trabajos pasivos son los trabajos de vigilancia de varios tipos en los procesos industriales.

Trabajos de baja tensión TBTL): Dados por la combinación de bajas demandas y alto control. En tales trabajos el riesgo por tensión y enfermedad es más bajo que en el promedio. Empíricamente pocos trabajos; o mejor, situaciones de trabajo se encuentran en este tipo.

Trabajos activos (TA): Provienen de la combinación de altas demandas y alto control. Resultan de las situaciones exigentes, pero estimulantes sin riesgo particular de tensión psicológica o enfermedad. Las personas con trabajos activos son también activas en las actividades de tiempo libre. Ejemplos de trabajos activos suelen ser los encontrados entre los profesionales, así como en trabajadores por cuenta propia como los granjeros, pequeños y medianos campesinos.

El modelo "demanda - control - apoyo social" que define estos cuatro grandes grupos de ocupaciones en función de los niveles de demandas psicológicas y control, en donde, la situación de alta tensión laboral y el bajo control decisional, es la más negativa para la salud, denominado Job strain; mientras que, el trabajo activo conduce a un mayor aprendizaje y al desarrollo de un mayor rango de estrategias de afrontamiento y participación social. Adicionalmente, se presenta un doble efecto del apoyo social en el trabajo, siendo la situación más negativa para la salud la que se caracteriza por la combinación de alta tensión psicológica y bajo apoyo social, denominado iso-strain (Moncada S, Lorens C, Sánchez E, 2000).

\section{Factores de riesgos psicosociales}

Los factores psicosociales de riesgo en el trabajo se refieren a "interacciones entre el trabajo, medio ambiente, la satisfacción en el trabajo, las condiciones de organización y capacidades del trabajador, necesidades, cultura, consideraciones personales fuera del trabajo que, a través de percepciones y experiencias pueden influir en la salud y el rendimiento y la satisfacción en el trabajo" (Comité mixto OIT-OMS, septiembre de 1984).

Los riesgos psicosociales perjudican la salud de los trabajadores, causando estrés y a largo plazo enfermedades cardiovasculares, respiratorias, inmunitarias, gastrointestinales, dermatológicas, endocrinológicas, musculoesqueléticas y mentales. Son consecuencia de unas malas condiciones de trabajo, concretamente de una deficiente organización del trabajo (García S. 2011).

El modo de trabajo y la organización del mismo en el sector de la construcción llevan asociadas una serie de características que favorecen la aparición del estrés, tales como (Arteaga, 2010):

a) El exceso de horas continuadas de trabajo,

b) La realización de tareas inhabituales o extraordinarias,

c) El cansancio o fatiga,

d) Las posturas forzadas o la realización de sobreesfuerzos durante la tarea,

e) Trabajar muy rápidoy en ocasiones con instrucciones de trabajo inexistentes o inadecuadas.

El estrés se considera como un proceso interactivo en el que influyen los aspectos del trabajo (demandas psicológicas) y las características del sujeto o trabajador (recursos). Cuando las demandas superan los recursos del individuo, se produce una situación de estrés donde el individuo intentará generar más recursos para atender las demandas del trabajo (Merín Reig, Cano Vindel y Miguel Tobal, 1995, p. 118).

Existen tres formas de referirse a los aspectos psicosociales, como: factores psicosociales, factores psicosociales de riesgo y riesgos psicosociales, estos términos se han tratado de manera similar; sin embargo es necesario establecer su diferencia (Tabla 1) (Moreno, 2002).

Los factores psicosociales laborales son el conjunto de percepciones y experiencias del empleado; interacciones con el trabajo, su medio ambiente, satisfacción, necesidades, cultura y situación personal.

Los factores psicosociales de riesgo son las condiciones organizacionales que poseen elementos con probabilidad de afectar negativamente la salud física y psicológica del trabajador. Los riesgos psicosociales son situaciones laborales que tienen una alta probabilidad de dañar gravemente la salud de los trabajadores, física, social o mentalmente. 
La OMS, en el informe 571 del año 1975, clasifica los factores psicosociales del trabajo que son nocivos y que generan o favorecen la aparición de alteraciones de la salud según el tipo de trabajo, las condiciones de trabajo y la organización del trabajo (Tabla 1).

Tabla 1. Clasificación de los factores psicosociales del trabajo, según OMS, 1975)

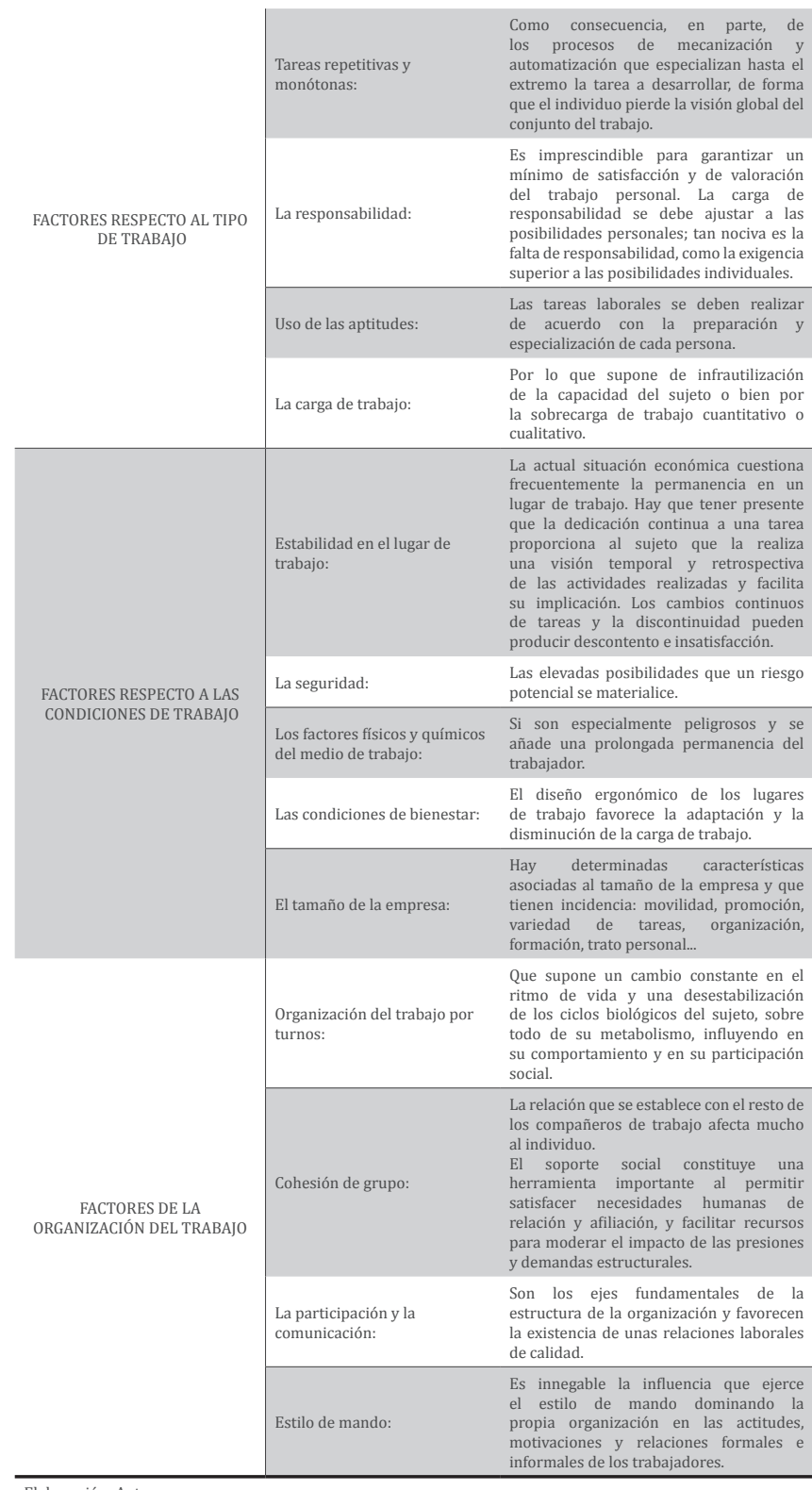

Elaboración: Autor

Los principales efectos negativos del estrés laboral según (Doval et al. 2004, Efectos negativos del estrés en el trabajador), se resumen en la Tabla 2:
Tabla 2. Efectos negativos del estrés

\begin{tabular}{|c|c|c|}
\hline EFECTOS FISIOLÓGICOS & EFECTOS COGNITIVOS & EFECTOS MOTORES \\
\hline $\begin{array}{l}\text { - Aumento de la tasa } \\
\text { cardiaca. } \\
\text { - Tensión muscular. } \\
\text { - Dificultades para } \\
\text { respirar. }\end{array}$ & $\begin{array}{l}\text { - Preocupaciones } \\
\text { - Dificultad para la toma } \\
\text { de decisiones. } \\
\text { - Sensación de confusión. }\end{array}$ & $\begin{array}{l}\text { - Hablar rápido } \\
\text { - Temblores } \\
\text { - Tartamudeo }\end{array}$ \\
\hline
\end{tabular}

Sobre la salud, el estrés puede provocar los siguientes efectos Tabla 3:

Tabla 3. Efectos del estrés sobre la salud.

\begin{tabular}{c|c}
\hline ENFERMEDADES POR ESTRÉS AGUDO & PATOLOGÍAS POR ESTRÉS CRÓNICO \\
$\begin{array}{c}\text { •Úlcera por estrés. } \\
\text { • Estados de Shock. }\end{array}$ & $\bullet$ Dispepsia. \\
$\bullet$ Neurosis post traumática. & $\bullet$ Anstritis. \\
& $\bullet$ Accidentes. \\
& $\bullet$ Frustración. \\
\hline
\end{tabular}

Fuente: Villalobos 1999, Enfermedades por Estrés

Los efectos del estrés sobre la organización, se pueden resumir en los siguientes (Villalobos 1999, Efectos del estrés sobre la organización):

1. Absentismo.

2. Rotación o fluctuación del personal.

3. Disminución del rendimiento físico.

\section{RESULTADOS}

Del personal que participó en el estudio (150 trabajadores) el $87 \%$ corresponde al género masculino y el $13 \%$ al género femenino, con un promedio de edad de 38 años. El reactivo utilizado arrojó un factor de confiabilidad según el alfa de Cronbach de 0,908, y un coeficiente de confiabilidad rk20 de 0,89 .

En tabla 4, se presenta los grupos de personas según el tipo de trabajo, en donde se aprecia que el 35 (23,3\%) trabajadores se encuentra expuestos a un trabajo de alta tensión laboral, 41 o $(27,3 \%)$ tiene un trabajo pasivo, 30 equivalente a $(20,0 \%)$ tiene un trabajo de baja tensión laboral y 44 o (29,3\%), se encuentra bajo un trabajo activo.

Tabla 4. Tipos de trabajo de acuerdo al modelo demanda-control de Karasek.

\begin{tabular}{l|c|cc}
\multicolumn{1}{c}{ TIPO DE TRABAJO } & \multicolumn{3}{c}{ EXPUESTOS } \\
\hline Trabajo de alta tensión laboral & TATL & 35 & $23 \%$ \\
\hline Trabajo pasivo & TP & 41 & $27 \%$ \\
\hline Trabajo de baja tensión laboral & TBTL & 30 & $20 \%$ \\
\hline Trabajo activo & TA & 44 & $29 \%$ \\
\hline Fuente: ator
\end{tabular}


La tabla 5 muestra el tipo de trabajo por sexo, en donde 20 $(13,3 \%)$ fueron mujeres y $130(86,7 \%)$ fueron hombres. El $40,0 \%$ de mujeres y el $20,8 \%$ de hombres, se encuentran expuestos en un trabajo de alta tensión laboral o trabajo estresante.

Tabla 5. Tipos de trabajo de acuerdo al modelo demandacontrol de Karasek por sexo.

\begin{tabular}{l|cccc|cccc|cc}
\multicolumn{3}{c}{ SEXO } & & \multicolumn{10}{c}{ TIPO DE TRABAJO } \\
\hline Hombre & 130 & $86,7 \%$ & 27 & $20,8 \%$ & 39 & $30,0 \%$ & 27 & $20,8 \%$ & 37 & $28,5 \%$ \\
Mujer & 20 & $13,3 \%$ & 8 & $40,0 \%$ & 2 & $10,0 \%$ & 3 & $15,0 \%$ & 7 & $35,0 \%$ \\
\hline TOTAL & 150 & $100 \%$ & 35 & & 41 & & 30 & & 44 & \\
\hline
\end{tabular}

En cuanto a los grupos de edad, la Tabla 6 muestra que 35 trabajadores están expuestos a un trabajo de alta tensión laboral con riesgo de estrés y enfermedad física, de los cuales 6 trabajadores que corresponden al 30,0\% (de 20) corresponden a mayores de 50 años; 8 trabajadores que corresponde al 24,2\% (de 33), están comprendidos en edades de 40 a 49 años, evidenciándose un aparente incremento en el riesgo en los grupos de mayor edad.

Tabla 6. Tipos de trabajo de acuerdo al modelo demandacontrol de Karasek por edad.

\begin{tabular}{|c|c|c|c|c|c|c|c|c|c|c|}
\hline RANGO DE EDAD & \multicolumn{2}{|c|}{ EDAD } & & \multicolumn{2}{|r|}{$\mathrm{TP}$} & \multicolumn{2}{|c|}{ TBTL } & \multicolumn{2}{|c|}{$\mathrm{TA}$} \\
\hline Mayor de 50 años & 20 & $13,3 \%$ & 6 & $30,0 \%$ & 8 & $40,0 \%$ & 0 & $0,0 \%$ & 6 & $30,0 \%$ \\
\hline De 40 a 49 años & 33 & $22,0 \%$ & 8 & $24,2 \%$ & 12 & $36,4 \%$ & 9 & $27,3 \%$ & 4 & $12,1 \%$ \\
\hline De 30 a 39 años & 41 & $27,3 \%$ & 8 & $19,5 \%$ & 12 & $29,3 \%$ & 11 & $26,8 \%$ & 10 & $24,4 \%$ \\
\hline Menor de 30 años & 56 & $37,3 \%$ & 13 & $23,2 \%$ & 9 & $16,1 \%$ & 10 & $17,9 \%$ & 24 & $42,9 \%$ \\
\hline & 150 & & 35 & & 41 & & 30 & & 44 & \\
\hline
\end{tabular}

En lo relacionado con Apoyo Social en el trabajo, Figura 2, se aprecia que el $85 \%$ de la muestra tiene buen apoyo de sus mandos (residentes de obra, superintendentes, etc.), el $83 \%$ evidencia buen apoyo social entre compañeros de trabajo. Esto da como resultado que la dimensión total de apoyo social es buena y corresponde al 85\%. Este alto nivel de apoyo social hace que el riesgo de sufrir tensión psíquica y enfermedad física se reduzca pese a las demandas psicológicas a las que se encuentra expuesto el personal.

Figura 2. Dimensión de Apoyo Social.

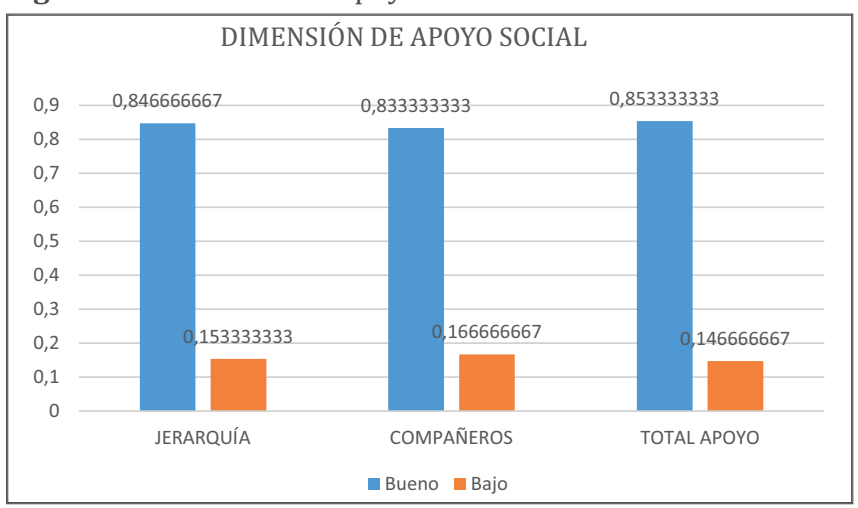

El modelo de estrés de Karasek, establece que cuando el trabajador se encuentra bajo una exposición de trabajo de alta tensión laboral y adicionalmente no cuenta con un buen nivel de apoyo social, incrementa el riesgo de alto estrés laboral denominado isostrain, que según el estudio corresponde al 7\% de la muestra. Es decir, el 7\% de la muestra tiene riesgo de alta tensión psicológica y enfermedad física (Figura 3).

Figura 3. Nivel de Isostrain.

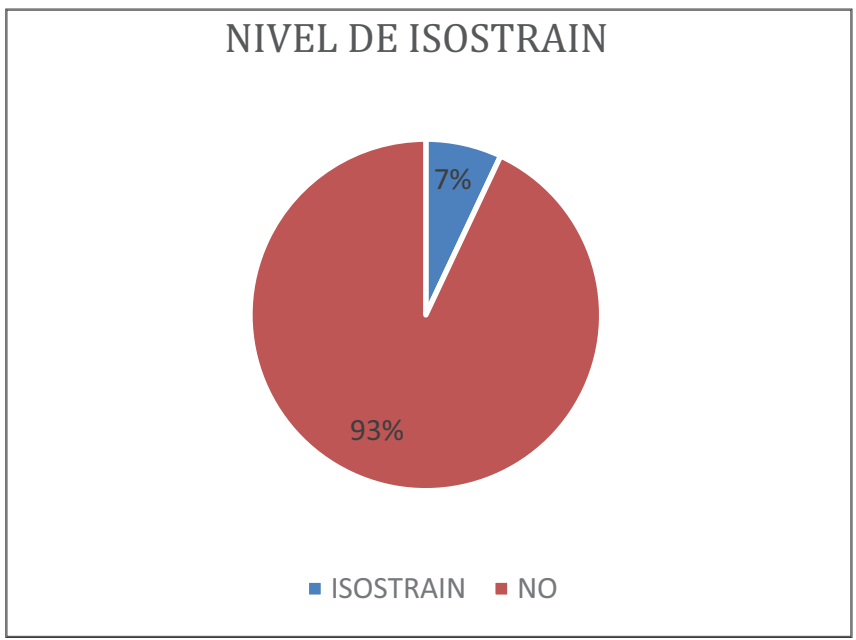

Del estudio se puede determinar las siguientes conclusiones:

1. Existe un bajo nivel de personas que ejecutan un trabajo de alta tensión laboral (23\%), y con riesgo de alta tensión psicológica y enfermedad física (Job stress).

2. El personal femenino tiene mayor riesgo de presentar un trabajo de alta tensión laboral (40\%).

3. Los trabajadores con edad mayor a 40 años tiene el más alto grado de tensión psicosocial.

4. Un 7\% de la población se encuentra en la etapa del trabajo de alta tensión laboral y bajo apoyo social, denominado isostrain, con un alto riesgo de tensión mental y enfermedad física.

5. De la muestra analizada, se concluye que existe trabajadores expuestos a niveles de alta tensión psicológica o nivel de estrés.

\section{RECOMENDACIONES}

1. Realizar un estudio posterior que incluya una encuesta de niveles de salud para determinar la relación existente entre las altas tensiones psicológicas y los síntomas que puede estar padeciendo el personal.

2. Aplicar programas de control y reducción del estrés laboral. 


\section{REFERENCIAS BIBLIOGRÁFICAS}

\section{Books (Libros):}

1. De la Cruz Rubio María (2010). Manual de riesgos psicosociales. El estrés y el síndrome de Burnout. CEP. Madrid, Pág. 18.

2. Moreno Bernardo, Báez Carmen. Factores y riesgos psicosociales, formas, consecuencias, medidas y buenas prácticas. Madrid. Pág. 4 - 22

3. Velázquez Manuel (2008). Impacto laboral del estrés. Lettera publicaciones. España.

4. Llaneza Javier (2009). Ergonomía y psicología aplicada. Lex Nova. España.

5. Ruiz-Frutos Carlos, García Ana (2007). Salud Laboral. Masson. España.

6. Peiró José (1993). Control del estrés laboral. Edutema. Madrid.

7. Peiró José (1993). Desencadenantes del Estrés Laboral. Edutema. Madrid.

\section{Periodicals (Artículos de revista):}

1. Román Jorge, (2003). Psicología aplicada a la salud y seguridad laborales. Cuba. Pág.11.

2. Vázquez Laura, (2010). Modelo demanda control e indicadores cardiovasculares en trabajadores de una empresa manufacturera, México.

3. Arribas Martín. Estrés relacionado con el trabajo (modelo demanda - control - apoyo social) y alteraciones en la salud: Una revisión de la evidencia existente. Madrid 2007. Pág. 169.

4. Gómez-Ortíz Viviola, Moreno Lorena, (2009). Factores psicosociales del trabajo (demanda-control y desbalance esfuerzo recompensa), salud mental y tensión arterial: un estudio con maestros escolares en Bogotá, Colombia.

5. Peña Pulido Antonio, otros, (2006). Valoración psicosocial mediante el uso de los modelos de estrés laboral de DemandaControl y desequilibrio Esfuerzo-Recompensa, España.

6. López Blanca, Osca Amparo (2010). El Papel del modelo demanda-control-apoyo social en la salud de los trabajadores de la construcción.

7. Gil-Monte Pedro (2012). Riesgos psicosociales en el trabajo y salud ocupacional.

8. Moncada S. Llorens C, Sánchez E, (2000). Factores psicosociales: la importancia de la organización del trabajo para la salud de las personas. Pág. 9. 